\title{
Alpha-power in electroencephalography as good outcome predictor for out-of-hospital cardiac arrest survivors
}

\section{Min-Jee Kim}

Asan Medical Center, Ulsan University College of Medicine

Youn-Jung Kim

Asan Medical Center, Ulsan University College of Medicine

Mi-Sun Yum ( $\square$ misun.yum@gmail.com )

Asan Medical Center, Ulsan University College of Medicine

Won Young Kim

Asan Medical Center, Ulsan University College of Medicine

\section{Research Article}

Keywords: Out-of-hospital cardiac arrest, Electroencephalography, Alpha frequency, Power spectral density

Posted Date: February 7th, 2022

DOI: https://doi.org/10.21203/rs.3.rs-1065871/v2

License: (c) (i) This work is licensed under a Creative Commons Attribution 4.0 International License. Read Full License 


\section{Abstract}

This study aimed to identify the quantitative EEG biomarkers for predicting good neurologic outcomes in OHCA survivors treated with targeted temperature management (TTM) using power spectral density (PSD), event-related spectral perturbation (ERSP), and spectral entropy (SE). This observational registrybased study was conducted at a tertiary care hospital in Korea using data of adult nontraumatic comatose OHCA survivors who underwent standard EEG and treated with TTM between 2010 and 2018. Good neurological outcome at 1 month (Cerebral Performance Category scores 1 and 2) was the primary outcome. The linear mixed model analysis was performed for PSD, ESRP, and SE values of all and each frequency band. Thirteen of the 54 comatose OHCA survivors with TTM and EEG, 13 were excluded due to poor EEG quality or periodic/rhythmic pattern, leaving 41 patients for analysis. The median time to EEG was $21 \mathrm{~h}$, and the rate of the good neurologic outcome at 1 month was $52.5 \%$. The good neurologic outcome group was significantly younger and showed higher PSD and ERSP and lower SE features for each frequency than the poor outcome group. After age adjustment, only the alpha-PSD was significantly higher in the good neurologic outcome group ( $1.13 \pm 1.11$ vs. $0.09 \pm 0.09, p=0.031)$ and had best performance with 0.903 of the area under the curve for predicting good neurologic outcome. Alpha-PSD best predicts good neurologic outcome in OHCA survivors and is an early biomarker for prognostication. Larger studies are needed to conclusively confirm these findings.

\section{Introduction}

More than 250,000 people in the USA and Europe annually experience out-of-hospital cardiac arrest $(\mathrm{OHCA})^{1,2}$ with only $8.3 \%$ of survivors having a favorable neurological outcome and being eligible for discharge ${ }^{2}$. Hypoxic-ischemic brain injury after the decision to withdraw life-sustaining therapy (WLST) is the leading cause of mortality, and accurate prediction of neurological outcomes is of utmost importance to guide the nature and duration of therapeutic interventions ${ }^{3,4}$. Recent recommendations proposed the strategy for predicting the neurological outcome based on a multimodal approach using clinical examination, electrophysiology, biomarkers, and brain imaging ${ }^{5-7}$.

Electroencephalography (EEG) is the most widely used and easily accessible tool to predict prognosis and detect subclinical seizures after cardiac arrest ${ }^{8}$. Although the standardized critical care EEG terminology proposed by American Clinical Neurophysiology Society (ACNS) and the standard EEG classification (highly malignant, malignant, and benign pattern), its clinical usability is limited by poor reproducibility and low inter-rater reliability even among experts ${ }^{9-16}$. Some specific EEG patterns or features were suggested for prognostication after cardiac arrest ${ }^{17,18}$, and various confounders including examination timing, the patient's condition, and different sedatives or anesthetics used in these patients interfere with the objective identification of these features by visual inspection ${ }^{18,19}$. These limitations encourage the development of quantitative EEG biomarkers for neurologic outcome prediction in $\mathrm{OHCA}$ instead of expert interpretation. 
To quantify the background EEG with standard recording, we analyzed the power, variability and randomness of each frequency band using the power spectral density (PSD), event-related spectral perturbation (ERSP), and spectral entropy (SE) in patients with OHCA treated with targeted temperature management (TTM). Finally, readily available and accurate quantitative biomarker for good outcomes was proposed with optimal cutoff values.

\section{Results}

Among 54 nontraumatic OHCA survivors who received TTM and underwent EEG, two and 11 patients were excluded due to poor ECG quality and periodic or rhythmic pattern, respectively. Thus, 41 patients were ultimately included in this study. Patients were categorized into the good $(n=21,52.5 \%)$ and poor ( $n$ $=20,47.5 \%)$ neurological outcome groups, respectively.

\section{Demographic and clinical characteristics of patients}

The demographic and clinical characteristics of the patients are summarized in Table 1. The patients in the good neurological outcome group were younger (mean, 51.9 vs. 65.2 years, $P=0.012$ ) and more frequently male $(85.7 \%$ vs. $50.0 \%, P=0.014)$. The patients in the good neurological outcome group also showed higher frequency of witnessed arrest ( $85.6 \%$ vs. $50.0 \%, P=0.014)$, initial shockable rhythm $(85.7 \%$ vs. $30.0 \%, P<0.001)$, and shorter resuscitation duration (median, 11.0 vs. $34.0 \mathrm{~min}, P=0.022$ ).

\section{Visual analysis of extracted EEGs}

Figure 1 shows examples of visual EEG analysis. Both patients in the good (right) and poor (left) neurologic outcome group showed symmetric, attenuated (all activities $<20 \mu \mathrm{V}, 100 \%$ of record) background activities over whole record. There was no stage-ll sleep transients or periodic rhythm. The dominant frequency and amplitude of background activities were difficult to differentiate between both patients in each group by visual analysis.

\section{Quantitative analysis of extracted EEGs according to background frequency}

PSD, ERSP, and SE were compared between the patients in the good and poor neurologic outcome groups to quantify the power, variability, and randomness of each frequency band (Table 2 and Figure 2). All and each frequency band-PSD of patients with good neurologic outcomes were significantly higher compared to that with poor neurologic outcomes (Table 2 and Figure 2A). Except for the delta frequency band, all and the rest frequency band-ERSP of the patients in the poor neurologic outcome group were lower than the patients in the good neurologic outcome group with statistical significance (Table 2 and Figure 2B). Moreover, all and each frequency band-SE of the patients with poor neurologic outcomes were significantly higher than patients with good neurologic outcomes (Table 2 and Figure 2C). Only alphaPSD was significantly higher in patients with good neurologic outcomes than those with poor neurologic outcomes after adjusting the age of each patient (Table 2 and Figure 2A).

\section{Useful biomarkers for predicting neurologic outcomes}


Among three values of all and each frequency band, the alpha-PSD was the most reliable biomarker to differentiate the neurologic outcomes with the area under the curve (AUC) of 0.903 and $p$ value $<0.001$. The alpha-PSD cutoff values of the averaged C3 and C4 electrodes were 0.14 with $82.4 \%$ sensitivity and $80.5 \%$ specificity or 0.20 with $72.4 \%$ sensitivity and $87.0 \%$ specificity, respectively (Figure 3 ).

\section{Discussion}

In this study, we quantified the power, variability, and randomness of each frequency band in OHCA survivors with routine EEG to investigate the factor that can differentiate the good and poor neurological outcomes. We identify that the alpha-PSD was most reliable biomarker to indicate good neurologic outcomes with an AUC of 0.903 .

Beyond the accurate description using Standardized Critical Care EEG Terminology, appropriate interpretation of these descriptions is essential for precise prognostication of neurologic outcomes in patients with OHCA. Among various background patterns defined in the Standardized Critical Care EEG Terminology, we found the dominant background frequency with alpha and theta waves were a powerful predictors (adjusted OR for favorable neurologic outcomes of 13.030 ) with high sensitivity (86.21\%) and negative predictive value $(91.30 \%)$ in our previous study ${ }^{20}$. However, the interrater variability is a challenging issue for neurologists and emergency physicians to accurately predict neurologic outcome and the agreement strength is decreased especially for background frequency interpretation ${ }^{21}$. In fact, the visual analysis of the EEG in this study was also difficult to show the differences of predominant frequency between patients with poor and good neurologic outcomes even with time expansion with bandpass filter (Figure 1). Thus, we focus the quantitative analysis to identify the power and dynamic changes of background brain oscillation according to each frequency band.

The brain has continuous neuronal oscillations even in resting-state and time-related variabilities and can reflect the subclinical brain dynamics during consciousness or unconsciousness. The ERSP has a strong ability to detect the time-related shift of the spectral powers from the baseline in a specific frequency ${ }^{22-}$ 25 . Further, this analysis allows extracting information about the time course of power changes in each frequency under specific tasks including auditory stimuli, attention, and cognitive processes ${ }^{26,27}$ and hyperactive state of the brain with seizure or epileptic spasms in previous studies ${ }^{28,29}$.

Under steady-state condition, the neuronal firing is constantly occurred to transfer the information between neuron, and a synchronous state is maintained. The weakly correlated action potentials of individual neurons, for any reason, can lead to the asynchronous state and increase randomness ${ }^{30-32}$, entropy. The entropy algorithms have already been widely used in analyzing depth of anesthesia 33,34 and detecting seizures ${ }^{35,36}$.

To identify the time-related variability and randomness of power in each frequency band, we calculate the ERSP and SE in this study, respectively. No significant differences of ERSP and SE in all frequencies between the groups after age adjustment indicated that the patients with OHCA had too low brain 
activities to measure the variability or randomness without external stimulation due to acute neuronal damage itself or treated sedatives.

The PSD analysis is a method for quantitatively evaluating the power distribution of target frequency ranges. The low amplitude of background activity of $\langle 20 \mu \mathrm{V}$ in the entire channels, including suppressed or attenuated EEG pattern, is classified as highly malignant or malignant EEG that predicts a poor neurologic outcome ${ }^{12}$. In these context, we quantify the background power of each frequency using PSD and identify that the powers of alpha frequency bands are increased in the good neurologic outcome group in line with previous studies ${ }^{12,37}$.

Regarding dominant background frequency, we are interested in alpha frequency band, which is related to the good neurological outcome in our previous and current studies ${ }^{20}$. Alpha oscillations are the salient features during resting or wakefulness, and the change of alpha frequency is related to aging, cognitive function, sensorimotor processing, sleep, and neurodegenerative disease including Alzheimer's disease ${ }^{38-40}$. A recent study with OHCA patients reported that the unfavorable outcome group showed lower power spectra in around $10 \mathrm{~Hz}$ alpha frequency compared with the favorable outcome group ${ }^{41}$. Furthermore, another study found that the prominent alpha peaks were presented in patients with minimally conscious states and in none of the comatose patients ${ }^{42}$. Consistently, the power of alpha frequency is higher in the good outcome group than in the poor outcome group after adjustment by age and the most reliable biomarker to predict neurologic outcomes in patients with OHCA.

To our knowledge, this is first report that quantify the three characteristics of the dominant background frequency to predict neurologic outcomes in OHCA patients. The findings of the current study suggest that alpha-PSD can be used as simple and reliable biomarkers for prognostication of neurologic outcomes. The results of the current study should be interpreted in the context of the following limitations. The retrospective study design and associated selection bias may have influenced the results. Moreover, the limited number of patients may have contributed to the lack of significance of some results of the current study. Another potential concern is the EEG timing and influence of sedative agents during EEG recording. However, the use of sedative agents followed the postcardiac arrest care protocol in this experienced tertiary center, and a relative homogenous standard routine EEG protocol was used. In addition, the cohort of the current study is not complicated by the WLST issue due to forbidden by law in South Korea during the study period.

Considering the poor interrater agreement and reproducibility of the visual interpretation of standard intermittent EEG, the quantitative analysis method with PSD, ERSP, and SE feature extraction presented in this study suggests alpha-PSD as early prognostic tools for neurologic outcome in patients with OHCA. Further larger studies are warranted to validate the predictive value of the alpha-PSD of early standard intermittent EEG proposed in this study.

\section{Methods}




\section{Study design and patients}

This retrospective, observational, registry-based cohort study was conducted at the emergency intensive care unit (ICU) of a tertiary care university-affiliated teaching hospital in Korea. Data were extracted from the OHCA registry containing prospectively collected data of consecutive patients with OHCA since January $2010^{43}$. The Institutional Review Board of the University of Ulsan College of Medicine, Seoul, Korea, reviewed and approved the study protocol (no. 2019-1883). Informed consent was waived due to the retrospective nature of the study by Institutional Review Board of the University of Ulsan College of Medicine, Seoul, Korea (no. 2019-1883).

This study included patients with successfully resuscitated nontraumatic OHCA >18 years old and treated with TTM due to neurologic impairments after the return of spontaneous circulation (ROSC) between January 2014 and December 2018. Routine EEG was recorded in the first $48 \mathrm{~h}$ after ROSC. All patients were comatose during EEG recording and TTM. We excluded patients with poor EEG quality which is impossible to extract the representative 5-min EEG or periodic/rhythmic EEG pattern. All patients were followed up to 1 month after cardiac arrest with neurologic assessment using the Cerebral Performance Category (CPC) score. The primary outcome of this study was a good neurologic outcome at 1 month, defined as CPC scores 1 and 2.

\section{Management And Data Collection}

All patients were treated according to the current advanced cardiac life support guidelines ${ }^{44,45}$. TTM was performed for all unconscious patients using Arctic Sun Energy Transfer Pad (Medivance Corp., Louisville, CO, USA), and the target temperature $\left(33^{\circ} \mathrm{C}\right.$ or $\left.36^{\circ} \mathrm{C}\right)$ was maintained for $24 \mathrm{~h}$. Patients were rewarmed at a rate of $0.25^{\circ} \mathrm{C} / \mathrm{h}$ after $24 \mathrm{~h}$, following maintained normothermia until $72 \mathrm{~h}$ from ROSC. The temperature was monitored using an esophageal temperature probe. A combination of propofol, midazolam, fentanyl, remifentanil, and morphine was used for sedation and analgesia. A neuromuscular blocking agent was administered to control shivering if necessary. Patients with seizure activity on EEG were treated with valproate or levetiracetam. All patients received standard intensive care according to institutional protocol. WLST was legally prohibited in South Korea until August $2017^{46}$, and none of the patients in the current study underwent WLST.

Demographic and clinical data, including age, sex, previous medical history, resuscitation profiles such as the presence of a witness on collapse, initial documented rhythm, and resuscitation duration) were obtained.

\section{Eeg Recording And Preprocessing}

Standard EEG examination was started during TTM after ICU admission. The 30-min scalp EEG was recorded by the Stellate EEG system from 21 electrodes that were placed according to the international 10-20 electrode system (Fp1-2, F7-8, T7-8, P7-8, F3-4, C3-4, P3-4, 01-2, Fz, Cz, and Pz) with a sampling rate of 200 and $0.1 \mathrm{~Hz}$ high-pass filter. EEGs were initially reviewed to select clean data without apparent 
artifacts or muscle activities, and 5 min of each artifact-free resting-state EEG were collected. The extracted EEGs were extended temporally, and $0.5,15$, and $30 \mathrm{~Hz}$ high-pass filters were used to differentiate visible background amplitude and frequency, including beta $(14-30 \mathrm{~Hz})$ and gamma (30$100 \mathrm{~Hz}$ ) with sensitivities of 10 and $5 \mu \mathrm{V} / \mathrm{mm}$, respectively (Figure 1). Before quantitative analysis, the data were preprocessed using the EEGLAB toolbox of MATLAB 2020a. The $0.5-\mathrm{Hz}$ high-pass and $60-\mathrm{Hz}$ notch filters were used to remove the alternating current at $60 \mathrm{~Hz}$, and independent component analysis was applied using runica (), plugged in EEGLAB, to remove artifacts. Finally, the extracted 5 min of EEG data of each patient were divided into 10 epochs (30-s duration) per patient. The EEG from the C3 and C4 electrodes were selected for data extraction to reduce the muscle and eyeball movement artifacts.

\section{Spectral Power Analysis (Power Spectral Density)}

The PSD estimated the power of each frequency band directly from the signal itself. The PSD (log) was computed by Welch power spectral estimation for each patient with a sampling rate $(200 \mathrm{~Hz})$ and $15-\mathrm{s}$ window size length segments in each 30 -s epoch. The average in each PSD frequency including delta $(0.5-4 \mathrm{~Hz})$, theta $(5-7 \mathrm{~Hz})$, alpha $(8-14 \mathrm{~Hz})$, beta $(15-29 \mathrm{~Hz})$, and gamma $(30-100 \mathrm{~Hz})$ extracted from the $\mathrm{C} 3$ and $\mathrm{C} 4$ electrodes of 10 epochs were calculated.

\section{Variability Analysis (Event-related Spectral Perturbation)}

ERSP ( $\log )$ was used to show dynamic brain changes with the zero point in each epoch set as the baseline. ERSPs were analyzed with fast Fourier transform and Hanning window tapering in each 30-s epoch. The mean baseline log power spectrum was subtracted to produce the baseline-normalized ERSP from each spectral estimate, and then deviations from baseline power were calculated. The ERSP formula for averaged estimates across data trials ( $n$ trials) is defined below:

$\operatorname{ERSP}(f, t)=\frac{1}{n} ? \stackrel{n}{k=1}\left|F_{k}(f, t)\right|^{2}$

where $F_{k}(f, t)$ is the spectral estimate of trial $k$ at frequency $f$ and time $t$. The averaged each frequency ERSP including delta $(0.5-4 \mathrm{~Hz})$, theta $(5-7 \mathrm{~Hz})$, alpha $(8-14 \mathrm{~Hz})$, beta $(15-29 \mathrm{~Hz})$, and gamma (30-100 $\mathrm{Hz}$ ) extracted from the $\mathrm{C} 3$ and $\mathrm{C} 4$ electrodes of 10 epochs were calculated.

\section{Randomness Analysis (Spectral Entropy)}

SE based on Shannon entropy in physics, quantifying the regularity/randomness of power spectrum during a given time, was used to establish one of the biomarkers for neurological prognosis after cardiac arrest in the recent study ${ }^{47}$. In effect, SE reflected the randomness of the power spectrum distribution. The greater SE represents the more uniform power spectral distribution ${ }^{48}$. Using previously calculated PSD $\left(P_{s d(f)}\right)$ via Welch's method, the normalized PSD, which was defined as the $P_{s d(f)}$ divided by the total power of each frequency band, was analyzed to obtain probability density function. 


$$
\operatorname{Psd}(f)=\frac{P s d(f)}{?_{f=a}^{f=b} P s d(f)}
$$

where $P s d(f)$ was the normalized PSD of $P_{s d(f)}$. SE was estimated based on the PSD within each frequency band: $a=0.5$ and $b=4$ for delta- $P s d(f) ; a=5$ and $b=7$ for theta- $P s d(f) ; a=8$ and $b=14$ for alpha-Psd(f); $a=15$ and $b=29$ for beta-Psd(f), and $a=30$ and $b=100$ gamma-Psd(f). The SE 0 was generated by using the following equation:

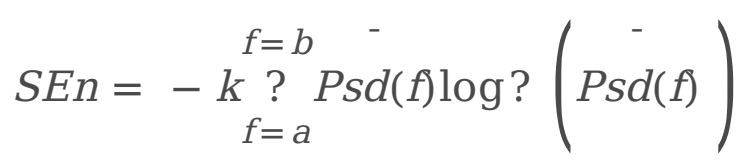

where $k=1$. The average of each frequency SE including delta $(0.5-4 \mathrm{~Hz})$, theta $(5-7 \mathrm{~Hz})$, alpha $(8-14$ $\mathrm{Hz})$, beta $(15-29 \mathrm{~Hz})$, and gamma $(30-100 \mathrm{~Hz})$ were extracted from $\mathrm{C} 3$ the $\mathrm{C} 4$ electrodes of 10 epochs were calculated.

\section{Statistical analysis}

Continuous variables are presented as median with interquartile range (IQR) due to the non-normal distribution using the Kolmogorov-Smirnov test. In addition, categorical variables are expressed as absolute numbers and percentages. The patients were categorized into two groups based on their CPC scores at 1 month: good neurologic (CPC 1 and 2) and poor neurologic (CPC 3-5) outcome groups. Comparisons of demographic and clinical characteristics between the good and poor neurologic outcome groups were performed using the Mann-Whitney U-test and chi-square test for continuous and categorical variables, respectively.

The linear mixed model analysis for PSD, ESRP, and SE values for all and each frequency band was performed to adjust the random effect of clustering from the epochs of the same patients at 10 timepoints and fixed effect of ages of each patient. Each variable was compared between the good and poor neurologic outcome groups, and receiver operating characteristic curves with $95 \%$ confidence intervals for good neurologic outcomes were analyzed to determine the cutoff values to predict neurologic prognosis. Sensitivity and specificity for good neurological outcomes at 6 months were calculated. All statistical analyses were performed using IBM SPSS for Windows, version 21.0 (IBM Corp., Armonk, NY, USA).

\section{Declarations}

\section{Acknowledgements:}

We thank all participants and their family members for taking part in this study.

\section{Data availability statement}


The datasets used and/or analysed during the current study available from the corresponding author on reasonable request

\section{Funding Source:}

This study was supported by a grant of the Korea Health Technology R\&D Project through the Korea Health Industry Development Institute (KHIDI), funded by the Ministry of Health \& Welfare, Republic of Korea (grant number: HI19C0481, HC20C0164).

In addition, this study was supported by the Basic Science Research Program, through the National Research Foundation of Korea (NRF-2021R1A2C2014304) funded by the Ministry of Science and ICT.

\section{Financial Disclosure and Conflicts of Interest}

All authors have disclosed that they do not have any conflicts of interest

\section{References}

1. Atwood, C., Eisenberg, M. S., Herlitz, J. \& Rea, T. D. Incidence of EMS-treated out-of-hospital cardiac arrest in Europe. Resuscitation 67, 75-80 (2005).

2. Mozaffarian, D. et al. Heart disease and stroke statistics--2015 update: a report from the American Heart Association. Circulation 131, e29-322 (2015).

3. Dragancea, I., Rundgren, M., Englund, E., Friberg, H. \& Cronberg, T. The influence of induced hypothermia and delayed prognostication on the mode of death after cardiac arrest. Resuscitation 84, 337-342 (2013).

4. Lemiale, V. et al. Intensive care unit mortality after cardiac arrest: the relative contribution of shock and brain injury in a large cohort. Intensive Care Med 39, 1972-1980 (2013).

5. Ben-Hamouda, N., Taccone, F. S., Rossetti, A. O. \& Oddo, M. Contemporary approach to neurologic prognostication of coma after cardiac arrest. Chest 146, 1375-1386 (2014).

6. Sandroni, C. et al. Prognostication in comatose survivors of cardiac arrest: an advisory statement from the European Resuscitation Council and the European Society of Intensive Care Medicine. Resuscitation 85, 1779-1789 (2014).

7. Nolan, J. P. et al. Outcome following admission to UK intensive care units after cardiac arrest: a secondary analysis of the ICNARC Case Mix Programme Database. Anaesthesia 62, 1207-1216 (2007).

8. Friberg, H. et al. Survey on current practices for neurological prognostication after cardiac arrest. Resuscitation 90, 158-162 (2015).

9. Hirsch, L. J. et al. The ACNS subcommittee on research terminology for continuous EEG monitoring: proposed standardized terminology for rhythmic and periodic EEG patterns encountered in critically ill patients. J Clin Neurophysio/ 22, 128-135 (2005). 
10. Tsuchida, T. N. et al. American clinical neurophysiology society standardized EEG terminology and categorization for the description of continuous EEG monitoring in neonates: report of the American Clinical Neurophysiology Society critical care monitoring committee. J Clin Neurophysio/ 30, 161-173 (2013).

11. Gaspard, N. et al. Interrater agreement for Critical Care EEG Terminology. Epilepsia 55, 1366-1373 (2014).

12. Beuchat, I., Solari, D., Novy, J., Oddo, M. \& Rossetti, A. O. Standardized EEG interpretation in patients after cardiac arrest: Correlation with other prognostic predictors. Resuscitation 126, 143-146 (2018).

13. Abend, N. S. et al. Interrater Agreement of EEG Interpretation After Pediatric Cardiac Arrest Using Standardized Critical Care EEG Terminology. J Clin Neurophysio/ 34, 534-541 (2017).

14. Sethi, N. K., Westhall, E., Rossetti, A. O. \& Cronberg, T. Standardized EEG interpretation accurately predicts prognosis after cardiac arrest. Neurology 87, 1631 (2016).

15. Abend, N. S. et al. Interobserver reproducibility of electroencephalogram interpretation in critically ill children. J Clin Neurophysio/ 28, 15-19 (2011).

16. Grant, A. C. et al. EEG interpretation reliability and interpreter confidence: a large single-center study. Epilepsy Behav 32, 102-107 (2014).

17. Young, G. B., McLachlan, R. S., Kreeft, J. H. \& Demelo, J. D. An electroencephalographic classification for coma. Can J Neurol Sci 24, 320-325 (1997).

18. Freeman, W. D. Continuous EEG in therapeutic hypothermia after cardiac arrest: prognostic and clinical value. Neurology 81, 855 (2013).

19. Westhall, E. et al. Interrater variability of EEG interpretation in comatose cardiac arrest patients. Clin Neurophysio/ 126, 2397-2404 (2015).

20. Kim, Y. J., Kim, M. J., Koo, Y. S. \& Kim, W. Y. Background Frequency Patterns in Standard Electroencephalography as an Early Prognostic Tool in Out-of-Hospital Cardiac Arrest Survivors Treated with Targeted Temperature Management. J Clin Med 9 (2020).

21. Westhall, E. et al. Standardized EEG interpretation accurately predicts prognosis after cardiac arrest. Neurology 86, 1482-1490 (2016).

22. Pfurtscheller, G. \& Lopes da Silva, F. H. Event-related EEG/MEG synchronization and desynchronization: basic principles. Clin Neurophysiol 110, 1842-1857 (1999).

23. Roach, B. J. \& Mathalon, D. H. Event-related EEG time-frequency analysis: an overview of measures and an analysis of early gamma band phase locking in schizophrenia. Schizophr Bull 34, 907-926 (2008).

24. Delorme, A. \& Makeig, S. EEGLAB: an open source toolbox for analysis of single-trial EEG dynamics including independent component analysis. J Neurosci Methods 134, 9-21 (2004).

25. Makeig, S., Debener, S., Onton, J. \& Delorme, A. Mining event-related brain dynamics. Trends Cogn Sci 8, 204-210 (2004). 
26. Tafuro, A., Ambrosini, E., Puccioni, O. \& Vallesi, A. Brain oscillations in cognitive control: A crosssectional study with a spatial stroop task. Neuropsychologia 133, 107190 (2019).

27. Chikara, R. K. \& Ko, L. W. Global Neural Activities Changes under Human Inhibitory Control Using Translational Scenario. Brain Sci 10 (2020).

28. Sowndhararajan, K., Kim, M., Deepa, P., Park, S. J. \& Kim, S. Application of the P300 Event-Related Potential in the Diagnosis of Epilepsy Disorder: A Review. Sci Pharm 86 (2018).

29. Kim, M. J., Yum, M. S., Yeh, H. R. \& Ko, T. S. Fast oscillation dynamics during hypsarrhythmia as a localization biomarker. J Neurophysiol 119, 679-687 (2018).

30. Uhlhaas, P. J. et al. Neural synchrony in cortical networks: history, concept and current status. Front Integr Neurosci 3, 17 (2009).

31. Carhart-Harris, R. L. The entropic brain - revisited. Neuropharmacology 142, 167-178 (2018).

32. Carhart-Harris, R. L. et al. The entropic brain: a theory of conscious states informed by neuroimaging research with psychedelic drugs. Front Hum Neurosci 8, 20 (2014).

33. Park, J. H. et al. Effect of depth of anesthesia on the phase lag entropy in patients undergoing general anesthesia by propofol: A STROBE-compliant study. Medicine (Baltimore) 99, e21303 (2020).

34. Shin, H. W. et al. Monitoring of anesthetic depth and EEG band power using phase lag entropy during propofol anesthesia. BMC Anesthesiol 20, 49 (2020).

35. Gao, X., Yan, X., Gao, P., Gao, X. \& Zhang, S. Automatic detection of epileptic seizure based on approximate entropy, recurrence quanti fi cation analysis and convolutional neural networks. Artif Intell Med 102, 101711 (2020).

36. Li, P. et al. Detection of epileptic seizure based on entropy analysis of short-term EEG. PLoS One 13, e0193691 (2018).

37. Backman, S. et al. Highly malignant routine EEG predicts poor prognosis after cardiac arrest in the Target Temperature Management trial. Resuscitation 131, 24-28 (2018).

38. Halgren, M. et al. The generation and propagation of the human alpha rhythm. Proc Natl Acad Sci U $S$ A 116, 23772-23782 (2019).

39. Mierau, A., Klimesch, W. \& Lefebvre, J. State-dependent alpha peak frequency shifts: Experimental evidence, potential mechanisms and functional implications. Neuroscience 360, 146-154 (2017).

40. Li, X., Yang, X. \& Sun, Z. Alpha rhythm slowing in a modified thalamo-cortico-thalamic model related with Alzheimer's disease. PLoS One 15, e0229950 (2020).

41. Kustermann, T. et al. Electroencephalography-based power spectra allow coma outcome prediction within 24 h of cardiac arrest. Resuscitation 142, 162-167 (2019).

42. Forgacs, P. B. et al. Dynamic regimes of neocortical activity linked to corticothalamic integrity correlate with outcomes in acute anoxic brain injury after cardiac arrest. Ann Clin Trans/ Neurol 4, 119-129 (2017).

43. Kim, Y. J. et al. The Role of Post-Resuscitation Electrocardiogram in Patients With ST-Segment Changes in the Immediate Post-Cardiac Arrest Period. JACC Cardiovasc Interv 10, 451-459 (2017). 
44. Peberdy, M. A. et al. Part 9: post-cardiac arrest care: 2010 American Heart Association Guidelines for Cardiopulmonary Resuscitation and Emergency Cardiovascular Care. Circulation 122, S768-786 (2010).

45. Callaway, C. W. et al. Part 8: Post-Cardiac Arrest Care: 2015 American Heart Association Guidelines Update for Cardiopulmonary Resuscitation and Emergency Cardiovascular Care. Circulation 132, S465-482 (2015).

46. Kim, Y. J. et al. Long-term neurological outcomes in patients after out-of-hospital cardiac arrest. Resuscitation 101, 1-5 (2016).

47. Tjepkema-Cloostermans, M. C., van Meulen, F. B., Meinsma, G. \& van Putten, M. J. A Cerebral Recovery Index (CRI) for early prognosis in patients after cardiac arrest. Crit Care 17, R252 (2013).

48. Kapucu, F. E. et al. Spectral Entropy Based Neuronal Network Synchronization Analysis Based on Microelectrode Array Measurements. Front Comput Neurosci 10, 112 (2016).

\section{Tables}

Table 1. Comparison of the clinical characteristics between the out-of-hospital cardiac arrest patients with good and poor neurologic outcomes at 1 month 


\begin{tabular}{|c|c|c|c|c|}
\hline Characteristics & $\begin{array}{l}\text { Total }(n= \\
41)\end{array}$ & $\begin{array}{l}\text { Good neurologic } \\
\text { outcome }(n=21)\end{array}$ & $\begin{array}{l}\text { Poor neurologic } \\
\text { outcome }(n=20)\end{array}$ & $\begin{array}{l}p \\
\text { value }\end{array}$ \\
\hline Age, years & $\begin{array}{l}58.4 \\
(17.3)\end{array}$ & $51.9(14.9)$ & $65.2(17.3)$ & 0.012 \\
\hline Male & $\begin{array}{l}28 \\
(68.3 \%)\end{array}$ & $18(85.7 \%)$ & $10(50.0 \%)$ & 0.014 \\
\hline \multicolumn{5}{|l|}{ Previous medical history } \\
\hline No comorbid disease & $\begin{array}{l}13 \\
(31.7 \%)\end{array}$ & $10(47.6 \%)$ & $3(15.0 \%)$ & 0.025 \\
\hline Hypertension & $\begin{array}{l}10 \\
(24.4 \%)\end{array}$ & $4(19.0 \%)$ & $6(30.0 \%)$ & 0.484 \\
\hline Diabetes mellitus & $7(17.1 \%)$ & $2(9.5 \%)$ & $5(25.0 \%)$ & 0.238 \\
\hline Congestive heart failure & $3(7.3 \%)$ & $1(4.8 \%)$ & $2(10.0 \%)$ & 0.606 \\
\hline Chronic kidney disease & $3(7.3 \%)$ & $0(0 \%)$ & $3(15.0 \%)$ & 0.107 \\
\hline \multicolumn{5}{|l|}{ Arrest characteristics } \\
\hline Presence of a witness & $\begin{array}{l}28 \\
(68.3 \%)\end{array}$ & $18(85.7 \%)$ & $10(50.0 \%)$ & 0.014 \\
\hline Bystander CPR & $\begin{array}{l}25 \\
(61.0 \%)\end{array}$ & $14(66.7 \%)$ & $11(55.0 \%)$ & 0.444 \\
\hline Initial shockable rhythm & $\begin{array}{l}24 \\
(58.5 \%)\end{array}$ & $18(85.7 \%)$ & $6(30.0 \%)$ & $<0.001$ \\
\hline No flow time, min & $\begin{array}{l}0.0(0.0- \\
5.0)\end{array}$ & $0.0(0.0-8.5)$ & $1.0(0.0-4.5)$ & 0.877 \\
\hline $\begin{array}{l}\text { Resuscitation duration, } \\
\text { min }\end{array}$ & $\begin{array}{l}18.0(7.5- \\
37.5)\end{array}$ & $11.0(6.5-24.5)$ & $34.0(11.0-41.5)$ & 0.022 \\
\hline $\begin{array}{l}\text { Time from ROSC to target } \\
\text { temperature, min }\end{array}$ & $\begin{array}{l}437 \\
(236.4)\end{array}$ & $423(189.5)$ & $453(284.0)$ & 0.696 \\
\hline $\begin{array}{l}\text { Time from ROSC to EEG, } \\
\text { hours }\end{array}$ & $\begin{array}{l}21.0 \\
(11.5- \\
37.0)\end{array}$ & $19.0(8.5-39.5)$ & $22.5(17.3-36.8)$ & 0.481 \\
\hline Treated sedative & $\begin{array}{l}41 \\
(100.0 \%)\end{array}$ & $21(100.0 \%)$ & $20(100.0 \%)$ & 1.000 \\
\hline Propofol & $\begin{array}{l}36 \\
(87.8 \%)\end{array}$ & 19 (90.5\%) & $17(85.0 \%)$ & 0.486 \\
\hline Midazolam & $6(14.6 \%)$ & $5(23.8 \%)$ & $1(5.0 \%)$ & 0.102 \\
\hline Fentanyl & $\begin{array}{l}37 \\
(90.2 \%)\end{array}$ & 19 (90.5\%) & $18(90.0 \%)$ & 0.481 \\
\hline
\end{tabular}


Remifentanil

16

$(39.2 \%)$

Morphine

18

$(43.9 \%)$

$8(38.1 \%)$

$8(40.0 \%)$

0.574

18

9 (42.9\%)

$9(45.0 \%)$

0.567

Values are expressed as median (interquartile ranges) or $n(\%)$ as appropriate.

CPR, cardiopulmonary resuscitation; ROSC, return of spontaneous circulation; EEG, electroencephalography.

Table 2. Comparisons of quantitative analysis using PSD, ERSP, and SE of each frequency between the out-of-hospital cardiac arrest patients with good and poor neurologic outcomes at 1 month

\begin{tabular}{|c|c|c|c|c|c|}
\hline \multicolumn{2}{|c|}{$\begin{array}{l}\text { Quantitative analysis } \\
\text { according to each frequency }\end{array}$} & \multirow{2}{*}{$\begin{array}{l}\begin{array}{l}\text { Good neurologic } \\
\text { outcome }(n=21)\end{array} \\
0.01 \pm 0.01\end{array}$} & \multirow{2}{*}{$\begin{array}{l}\begin{array}{l}\text { Poor neurologic } \\
\text { outcome }(n=20)\end{array} \\
0.01 \pm 0.00\end{array}$} & \multirow{2}{*}{$\begin{array}{l}p \\
\text { value } \\
0.002\end{array}$} & \multirow{2}{*}{$\begin{array}{l}\begin{array}{l}\text { Age- } \\
\text { adjusted } \\
p \text { value }\end{array} \\
0.900\end{array}$} \\
\hline PSD & All & & & & \\
\hline & Delta & $13.65 \pm 11.39$ & $3.48 \pm 4.64$ & $<0.001$ & 0.837 \\
\hline & Theta & 2. $79 \pm 3.04$ & $0.45 \pm 0.76$ & 0.002 & 0.793 \\
\hline & Alpha & $1.13 \pm 1.11$ & $0.09 \pm 0.09$ & $<0.001$ & 0.031 \\
\hline & Beta & $0.11 \pm 0.10$ & $0.02 \pm 0.01$ & $<0.001$ & 0.072 \\
\hline & Gamma & $0.00 \pm 0.00$ & $0.00 \pm 0.00$ & 0.038 & 0.650 \\
\hline \multirow[t]{6}{*}{ ERSP } & All & $4.42 \pm 0.38$ & $4.29 \pm 0.31$ & 0.004 & 0.295 \\
\hline & Delta & $4.38 \pm 0.39$ & $4.43 \pm 0.71$ & 0.663 & 0.910 \\
\hline & Theta & $4.48 \pm 0.56$ & $4.31 \pm 0.57$ & 0.046 & 0.091 \\
\hline & Alpha & $4.45 \pm 0.49$ & $4.28 \pm 0.49$ & 0.028 & 0.653 \\
\hline & Beta & $4.33 \pm 0.26$ & $4.21 \pm 0.24$ & 0.001 & 0.091 \\
\hline & Gamma & $4.43 \pm 0.54$ & $4.29 \pm 0.43$ & 0.032 & 0.439 \\
\hline \multirow[t]{6}{*}{ SE } & All & $0.50 \pm 0.05$ & $0.56 \pm 0.11$ & 0.014 & 0.092 \\
\hline & Delta & $0.90 \pm 0.00$ & $0.91 \pm 0.02$ & 0.002 & 0.092 \\
\hline & Theta & $0.90 \pm 0.00$ & $0.91 \pm 0.02$ & 0.002 & 0.095 \\
\hline & Alpha & $0.80 \pm 0.01$ & $0.83 \pm 0.04$ & 0.002 & 0.071 \\
\hline & Beta & $0.66 \pm 0.02$ & $0.71 \pm 0.07$ & 0.003 & 0.063 \\
\hline & Gamma & $0.51 \pm 0.05$ & $0.57 \pm 0.11$ & 0.014 & 0.071 \\
\hline
\end{tabular}


PSD, Power spectral density; ERSP, Even-related spectral perturbation; SE, Spectral entropy

All frequency, $0.5-100 \mathrm{~Hz}$; delta frequency, $0.5-4 \mathrm{~Hz}$; theta frequency, $5-7 \mathrm{~Hz}$; alpha frequency, 8-14 Hz; beta frequency, $15-30 \mathrm{~Hz}$; and gamma frequency, $31-100 \mathrm{~Hz}$

\section{Figures}

\section{Figure 1}

Visual analysis of raw data.

Example of EEGs from patients with poor (left panel) and good (right panel) neurological outcomes. Top -EEG filtered with a $0.5-\mathrm{Hz}$ high-pass filter with a sensitivity of $10 \mu \mathrm{V} / \mathrm{mm}$. Middle-EEG filtered with a 14$\mathrm{Hz}$ high-pass filter with a sensitivity of $5 \mu \mathrm{V} / \mathrm{mm}$. Bottom-EEG filtered with a $30-\mathrm{Hz}$ high-pass filter with a sensitivity of $5 \mu \mathrm{V} / \mathrm{mm}$. The alpha-PSD values of this epoch in patients with poor (left panel) and good (right panel) neurologic outcomes were 0.06 and 3.07, respectively.

\section{Figure 2}

Scattered dots of (A) PSD, (B) ERSP, and (C) SEs of all and each frequency.

Small start, $p<0.05$, Large start, age-adjusted $p<0.05$ by linear mixed model. 


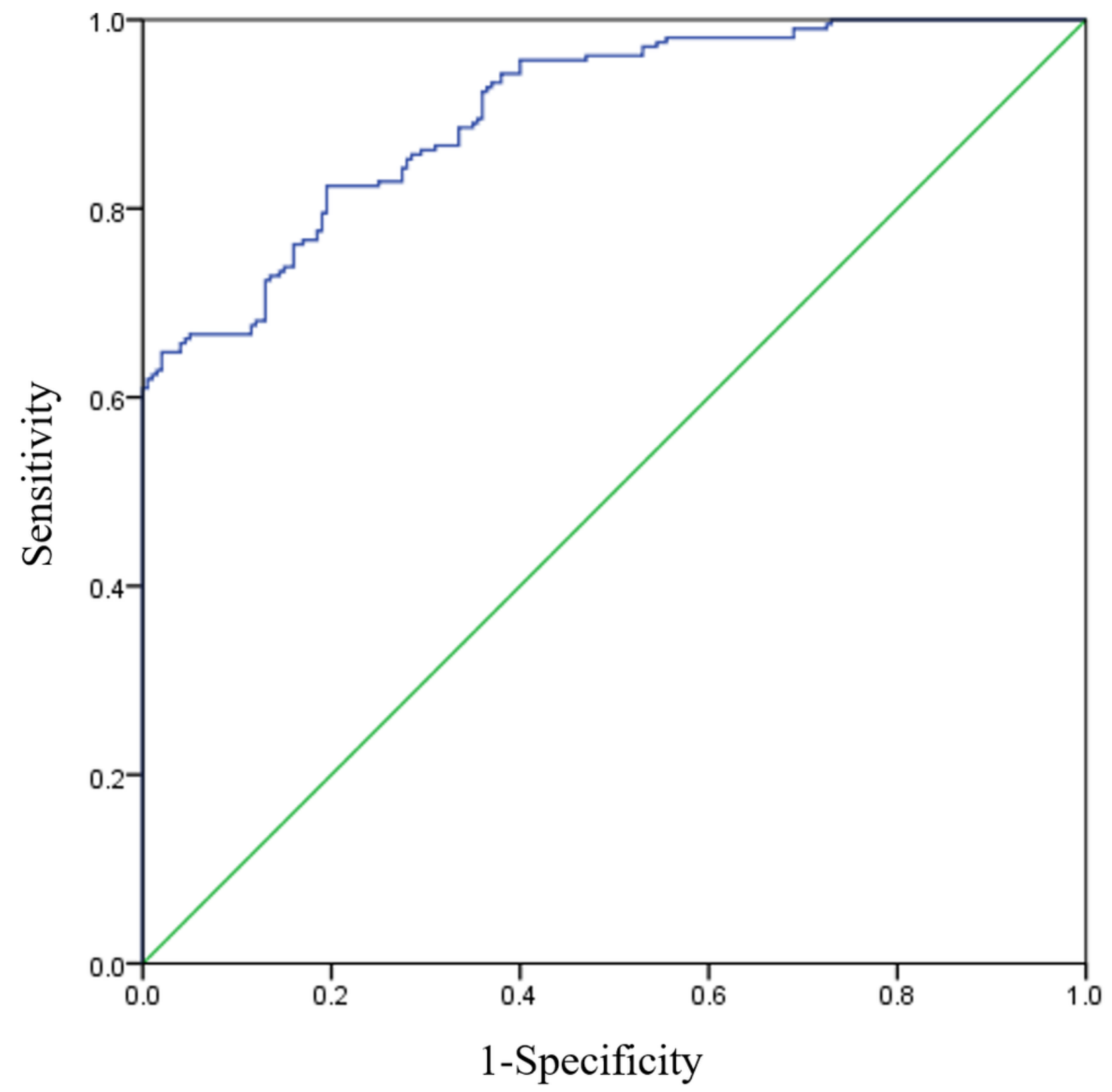

\begin{tabular}{|c|c|c|c|c|}
\hline $\begin{array}{c}\text { Threshold values } \\
\text { of alpha-PSD, } \\
\mathrm{dB}\end{array}$ & Sensitivity, \% & Specificity, \% & $\begin{array}{c}\text { Positive } \\
\text { predictive value, } \\
\%\end{array}$ & $\begin{array}{c}\text { Negative } \\
\text { predictive value, } \\
\%\end{array}$ \\
\hline 0.14 & 82.4 & 80.5 & 81.6 & 81.3 \\
\hline 0.20 & 72.4 & 87.0 & 85.4 & 75.0 \\
\hline
\end{tabular}

Figure 3

ROC curve and predictive values according to the cutoff of alpha-PSDs 